Mens

revue d'histoire intellectuelle de l'Amérique française

\title{
Jacques Dufresne. Après l'homme... le cyborg ? Sainte-Foy, Éditions MultiMondes, 1999. 178 p.
}

\section{Patrick Dionne}

Volume 3, numéro 2, printemps 2003

URI : https://id.erudit.org/iderudit/1024647ar

DOI : https://doi.org/10.7202/1024647ar

Aller au sommaire du numéro

\section{Éditeur(s)}

Centre de recherche en civilisation canadienne-française

\section{ISSN}

1492-8647 (imprimé)

1927-9299 (numérique)

Découvrir la revue

Citer ce compte rendu

Dionne, P. (2003). Compte rendu de [Jacques Dufresne. Après l'homme... le cyborg ? Sainte-Foy, Éditions MultiMondes, 1999. 178 p.] Mens, 3(2), 279-284.

https://doi.org/10.7202/1024647ar d'utilisation que vous pouvez consulter en ligne.

https://apropos.erudit.org/fr/usagers/politique-dutilisation/ 


\section{Jacques Dufresne. Après l'homme... le cyborg? Sainte-Foy, Éditions MultiMondes, 1999. 178 p.}

Magistrale par la lucidité de son analyse, brillante par l'universalité de son propos, cette cuvre du philosophe Jacques Dufresne se situe dans la lignée des grands écrits sur la question de l'homme à l'âge de la technique. Sa filiation intellectuelle avec les remarquables travaux de Jacques Ellul, d'Ivan Illich et de Gabriel Marcel nous confirme que nous sommes en présence d'un ouvrage important, rédigé par un homme de conviction, dont les vues pénétrantes ne manqueront pas de déplaire aux collectionneurs de lieux communs et aux dévoreurs de banalités.

Après l'bomme... le cyborg? est la réflexion critique d'un philosophe alarmé par la montée de la religion du progrès technique et par l'emprise graduelle qu'elle exerce sur le champ de la connaissance et de la pensée. Inquiétude légitime, quand on constate que les sociétés industrielles ont de plus en plus tendance à penser l'homme à partir de la machine et que, de ce fait, maintes activités humaines, technicisées à outrance, ressemblent de plus en plus à celles des machines. Il n'est pas plus rassurant de voir que cette conception techniciste de la réalité mène à la dégradation de l'idée de «valeur", qui se manifeste principalement, comme l'a noté Gabriel Marcel, lorsqu'on juge une personne à partir de critères infra-humains, ou si l'on préfère, inférieurs à l'humain. Or ces critères infra-humains, qui par définition ne devraient être utilisés que pour déterminer la valeur d'un objet matériel, sont paradoxalement les "valeurs » consacrées de la société industrielle : le rendement, l'efficacité, la productivité et la rentabilité. On observe déjà les ravages que provoquent ces critères d'évaluation quand on les applique à l'homme : isolement, misère, désespoir, suicide. Des démonstrations éclairées et rigoureuses s'avèrent donc nécessaires si nous voulons éviter que ne se poursuive la transposition 
effective de la mentalité techniciste sur l'homme, et si nous désirons que nous soient épargnées les horreurs du «meilleur des mondes ».

Le livre de Jacques Dufresne répond parfaitement à cette nécessité. L'auteur s'élève en effet contre cette conception de l'homme et du réel et craint le cas limite - et fort envisageable, car les scientifiques multiplient les recherches en ce sens (on clone déjà des animaux) et les timides débats éthiques ne parviennent pas vraiment à éveiller la conscience collective à l'urgence de la situation - de la création du cyborg, hybride monstrueux de l'être humain et de la machine:

Quand on m'apprend maintenant que je dois me réjouir de ressembler à une machine, si complexe soitelle, et que c'est à ce prix que je ferai le saut dans une espèce plus évoluée que l'homme-singe, cette promotion m'enlève mes derniers rêves. J'ai entrepris d'écrire ce livre avec l'espoir d'éloigner l'échéance de cette promotion, de moi-même d'abord, et des quelques amis de la sagesse éternelle qui voudront bien me lire. (p. 5)

Avec une clarté admirable, l'auteur parvient à renverser les illusions et les mythes engendrés par la religion du progrès technique, puis à redonner aux médias et à la connaissance médiate (ainsi qu'à la technique en général, implicitement) leur sens véritable en les réassignant à leur rôle, celui d'intermédiaires entre l'homme et la réalité. Quant à savoir si le livre retardera l'avènement du cyborg (ou du clonage humain, tout aussi rebutant), on ne peut que l'espérer...

Le premier chapitre relate l'histoire des métamorphoses de la connaissance en Occident, depuis le XIII ${ }^{e}$ siècle jusqu'à nos jours. Dufresne démontre que l'éclatement des médias et la notion de cyborg ne pouvaient advenir que dans un monde où les façons traditionnelles de penser la connaissance, mais aussi la liberté et la langue, étaient bouleversées. En s'inspirant 
de Gilbert Durand, l'auteur identifie trois désastres métaphysiques à l'origine de ce bouleversement. Le premier est la préférence que le Moyen Âge a accordé à Aristote au détriment de Platon (en privilégiant Averroès plutôt qu'Avicenne), ce qui aurait conduit la pensée occidentale à se couper de tout rapport direct à la transcendance. Pourtant, même si les penseurs du Moyen Âge avaient favorisé le platonisme plutôt que la philosophie d'Aristote, il n'est pas certain que cela aurait suffit à préserver la pensée occidentale d'une rupture de tout lien direct à la transcendance ; une argumentation plus ample aurait été bienvenue sur ce point. De même, peut-être qu'une discussion de la théorie de la pure nature (théorie qui considère la finalité naturelle de l'homme comme sa véritable fin dernière et qui, par là même, lui subordonne toute finalité surnaturelle) aurait enrichi cette section. La seconde catastrophe est la «substitution de la connaissance objective à la connaissance immédiate » (p. 20), amorcée par les penseurs du XVI siècle et achevée par ceux du XVIII", qui aurait amené l'homme à développer une vision mécaniste de l'univers et de lui-même. Le dernier désastre métaphysique est l'historicisme, cette doctrine qui donne un sens à l'histoire et qui fait du présent une ébauche grossière de l'avenir, c'est-à-dire une « chose » indéfiniment modifiable et sacrifiée au mythe du progrès.

Selon Dufresne, ces catastrophes métaphysiques ont entraîné le déclin de la connaissance immédiate, dont les moyens sont l'action et la contemplation. Il observe que les médias et la connaissance médiate se sont même substitués à la connaissance immédiate, que «les images sont désormais beaucoup plus importantes que les choses qu'elles représentent » (p. 3) et que l'homme en est venu à confondre la réalité avec l'image, jusqu'à ne plus être en mesure de les distinguer. Le péril ne vient pas de l'image elle-même, simple pont vers la connais- 
sance, mais de l'adoration de cette image, qui se nomme l'idolâtrie.

L'auteur analyse également les transformations de l'idée de liberté. Il dénonce notamment l'illusion que l'homme moderne entretient en s'imaginant que la multiplicité des choix qui s'offrent à lui le rendent plus libre que ses ancêtres. Or l'infinité des possibilités n'est pas un gage de liberté : c'est, affirme Dufresne, le désir de choisir qui est une garantie de liberté. Il montre d'ailleurs que la surabondance des possibilités finit par noyer ce qui est porteur de sens dans un flot d'insignifiances, et que cette idolâtrie du choix débouche sur une sorte "d'ignorance active » dépourvue de tout fondement moral : " [...] d'un univers moral où l'idéal était de vouloir le bien, on est passé à un univers où c'est le vouloir qui crée le bien. On ne choisit plus une chose parce qu'elle est bonne, la chose devient bonne parce qu'on la choisit. » (p. 34)

Dufresne nous convie aussi à combattre les maladies sémantiques iatrogènes, qui dénaturent les mots en les vidant de leur sens et qui éloignent l'homme du réel. En effet, les mots sont devenus aujourd'hui de pures conventions : ils sont "flottants », c'est-à-dire qu'ils subissent la contagion du relativisme des opinions et finissent par ne plus être en mesure de définir et même de signifier quoi que ce soit. Une publicité dégénérée comme celle de cette compagnie d'automobiles, qui dit le plus sérieusement du monde que "Driving is believing " (Conduire c'est croire), est un exemple frappant qui prouve les assertions de l'auteur.

L'auteur nous propose, dans le second chapitre, une réflexion normative sur la personne, la famille, l'école et la nation à l'âge de la technique, et nous explique comment ces réalités sont affectées par la prolifération des images. Selon lui, l'alexithymie (terme qui traduit une impuissance à faire correspondre un mot à un sentiment) est le «mal du siècle». Dia- 
gnostic on ne peut plus juste, dont les symptômes - partout visibles pour qui est le moindrement attentif — sont une incapacité à verbaliser ses émotions, un attachement obsessif aux détails, une atrophie de la créativité et une tendance à fuir ses problèmes dans l'action. Enfin, l'auteur nous lance des invitations à modifier nos comportements à l'égard des médias : il prône un jeûne médiatique et nous suggère de renouer avec l'écriture à la main, reflet de l'âme.

Dans le troisième chapitre (intitulé L'illusion et l'espérance), Dufresne répond à la question du choix initial entre l'homme et le cyborg (il se positionne pour l'homme), en étant pleinement conscient que l'humanité est parvenue à un temps de son histoire où la décision qu'elle prendra sera déterminante. Car ce que l'humanité risque, pour la première fois, c'est de s'enfermer dans un état de nature où elle ne peut que mourir asphyxiée. De la négation des fins surnaturelles de l'homme à la suppression des conditions qui disposent l'âme à vivre en rapport avec la transcendance, il n'y a plus, de nos jours, qu'un seul pas.

Dufresne retrace les origines millénaristes de la « démesure technique ", et procède à des distinctions capitales entre la vraie et la fausse transcendance pour couper court à toutes les illusions des apôtres du progrès technique qui souhaitent réaliser le paradis sur terre : «le ciel, le paradis chrétien, est en haut, non en avant : l'éternité est d'un autre ordre, c'est la ligne verticale qui symbolise la transcendance et non la ligne horizontale » (p. 166).

Finalement, cet ouvrage marque un point tournant de la philosophie d'expression française au Québec: Jacques Dufresne signe une œuvre personnelle, qui s'inspire en même temps de ces grands esprits que furent Simone Weil, Gustave Thibon, Ludwig Klages et Jacques Ellul et qu'il nous exhorte à fréquenter. Les œuvres de philosophie concrète n'abondent pas 
chez nous et celles de ce calibre se comptent sur les doigts d'une seule main. On sort de la lecture d'Après l'homme...le cyborg? avec la conviction que l'homme doit maitriser la technique avant d'être totalement dominée par elle. L'urgence de notre temps est de trouver des solutions au problème de la dépendance de l'homme à la technique. Nous avons besoin de solutions concrètes, collectives et durables afin que «l'homme assisté » retrouve sa liberté là où elle l'attend. À vrai dire, Après l'homme...le cyborg? s'avère un livre essentiel, actuel par son enracinement dans le temps, empreint d'éternité par son ouverture à la transcendance.

Gustave Thibon, dans Au soir de ma vie, écrivait que Jacques Dufresne était « un esprit aussi pénétrant qu’universel ». Nous en avons, encore une fois, la preuve éclatante.

Patrick Dionne

Bibliothèque, Couvent Saint-Albert-le-Grand

Bruce Curtis. The Politics of Population. State Formation, Statistics, and the Census of Canada, 1840-1875. Toronto, University of Toronto Press, 2001. 385 p.

Après nous avoir offert plusieurs excellents ouvrages et articles portant sur le développement du système d'éducation au Canada-Ouest après 1840, le sociologue Bruce Curtis s'intéresse maintenant au développement de la statistique canadienne construite à partir des recensements tenus de 1840 à 1875. Curtis apparait comme un des meilleurs présentateurs de la thèse de la «formation de l'État » au Canada. Cet ouvrage fait d'ailleurs ressortir toute la richesse de ce courant historiographique. Trop souvent présentée de manière méca- 